\title{
Molecular Detection of Virulence Factor Glycoprotein (Gp63) of Leishmania spp. in Phlebotomus Sand Flies
}

\author{
Hadi M. Hamza Al-Mayalii* ${ }^{\mathbb{D}}$, Ali Jawad Abdulaali Alyasiri² \\ ${ }^{1}$ Department of Biology, Education College, University of Al-Qadisiyah, Iraq \\ ${ }^{2}$ Department of Biology, Science College, Al-Muthanna University, Iraq
}

\begin{abstract}
Introduction: Gp63 is the major surface glycoprotein of Leishmania which is prevalent in the promastigote stage of Phlebotomus sergenti. Glycoprotein 63 (gp63) or leishmanolysin is a zinc-dependent metalloprotease found on the surface of Leishmania. It was initially discovered in 1980and described biochemically and genetically as a surface antigen expressed in promastigotes of Leishmania species, having a range of substrates including casein, albumin, fibrinogen, haemoglobin, and gelatin. On the surface of amastigote, GP63 is present at a very low level. Glycoprotein 63 is a zinc-dependent metalloprotease that is active in the $\mathrm{pH}$ range of 7-10, which is neutral to alkaline.

Materials and Methods: During the study, which was carried out in Al-Muthanna province from July 2017 to August 2018 , about 2550 sand fly samples including 719 males and 1633 females were collected. Three species of sand flies were recorded according to morphological features which included Phlebotomus papatasi, Phlebotomus sergenti, and Sergentomyia sintoni. The caught specimens showed that $P$. papatasi was the most prevalent species. Two primers were used for the diagnosis of Leishmania spp. using a nested PCR technique, which was designed in a previous study for 1250 samples of sand flies from 25 locations in the study area.

Results: Results showed that 13 samples were positive, containing DNA for the Leishmania parasite, and 12 samples were negative. Positive samples include 11 samples of $L$. major species and only 2 samples of $L$. tropica species. Additionally, the results showed that the $L$. major species was the dominant species in the study area. Positive samples (13) of sand flies had Leishmania parasite based on molecular diagnosis and virulence factor gene (Gp63) of Leishmania parasite was detected in sand flies using PCR method The results showed that virulence factor gene (Gp63) was detected on the surface of promastigote. This refers to the presence of Leishmania parasite in sand fly. The study is the first one which detected the presence of the virulence factors gene (GP63) of Leishmania in the body of sand flies.

Conclusion: This study revealed the possibility of diagnosing leishmaniasis by virulence factor gene (Gp63) in leishmania promastigotes.

Keywords: Leishmaniasis, Virulence factor, Gp63, Al-Muthanna province.
\end{abstract}

Received: December 9, 2020, Accepted: December 20, 2020, ePublished: December 30, 2020

\section{Introduction}

Phlebotomine sand flies (Diptera: Psychodidae) are hematophagous insects and vectors of leishmaniasis and other bacterial and viral diseases, for several species of Sandflies, are minor in size, with a linear measure of about $3 \mathrm{~mm}$. They are known for long bodies and wings that are covered with dense hair as well as long legs (1). They act as vectors of human and animal infections (2). There are over 800 species of sandflies in the world, 98 of which are vectors of human leishmaniasis. They include 42 Phlebotomus species in the Old World and 56 Lutzomyia species in the New World (3).

Phlebotomines, typically mentioned as Leishmania spp. vectors, are protozoan parasites that cause kala-azar and various kinds of human leishmaniasis (4).

Proteinases play significant roles in parasite invasion of host cells and immune system, movement over tissue, and inflammation stimulation inside the host (5). When it comes to infecting their own host for survival and prevalence, parasites always employ the most basic skill. Many different surface proteins, including glycoinositolphospholipids (GIPLs), can be used by Leishmania as possible virulence factors (6). Actually, it is known that Leishmania can utilize various surface proteins, recognized as potential virulence factors (e.g., GIPLs, lipophosphoglycan, cysteine protease, glycoprotein 63), to thwart the host macrophage defense system and therefore, permitting its survival and progression within the harsh environment of the phagolysosome (7).

The gp63 is the main surface glycoprotein of Leishmania, which is prevalent in promastigotes of Leishmania. Glycoprotein 63 (GP63), also called leishmanolysin, is 
a zinc-dependent metalloprotease which is present on the surface of Leishmania. It was discovered for the first time in 1980, as a significant surface antigen presented in promastigotes of Leishmania species. GP63 contains albumin, gelatin, casein, fibrinogen, and haemoglobin. GP63 is present on the amastigote. GP63 as a zincdependent metalloprotease could even be active in $\mathrm{pH}$ range of 7-10 (8).

In L. major, there are seven gp63 genes and they exhibit the stage-specific differences in their expression: five homologous tenderly repeated gp63 genes 1-5 are expressed in promastigotes only, a separated gene 6 is expressed in both promastigotes and amastigotes, while a gene 7 is expressed in stationary phase promastigotes and amastigotes. gp63 is abundantly expressed on the surface of the promastigote form, upregulated in infectious metacyclic promastigotes, and have a low but detectable expression level in the intracellular amastigote stage (9).

\section{Materials and Methods \\ Collection Sites}

This study was conducted in Al-Muthanna province from July 2017 to August 2018. Sand fly traps were placed in fields around their resting places in large numbers eight times a month (twice a week). The most visible sand break sites are animal pens, human houses, bird houses, leaf scraps, tree holes, or under rocks, and animal burrows, rock cracks, and between vegetation.

\section{Collection Methods}

Sticky Paper Traps

Sticky paper traps represent a technique for sand fly sampling by intercepting them instead of the attraction process. These traps are usually economical and easy to use in large numbers. Sticky traps are usually preferred to light traps to collect sand fly from indoors because they are unattractive. Traps were placed in the evening one hour before sunset and were collected after sunshine in next morning. The sandflies were collected from the traps using small forceps, washed with diluted detergent, and kept in $70 \%$ alcohol in the Eppendorf tubes with appropriate labeling until treatment and subsequent laboratory identification (10). They were selected to study their morphology and perform molecular diagnosis.

\section{Light Traps}

These traps are frequently utilized in investigations of sandfly fields. Sandflies are caught with tiny light traps. Sandflies were sampled from peri-domestic and agricultural fields using these traps. Traps should be placed at a height of 1.5-2 $\mathrm{m}$ from the ground level, preferably at least $10 \mathrm{~m}$ away from any external light source (6).

As a result, areas near other light sources, as well as spots exposed to high winds, sites near buildings containing animals, and areas exposed to industrial odors and smoke should be avoided. Furthermore, no light traps were utilized at home since light traps may attract flies from outside and may not represent real endophagic/ endophilic species $(2,11,12)$.

\section{Laboratory Examination}

The sand flies that have been caught were removed using a small brush or forceps. The sand flies were processed in a conventional way. The sandflies were thoroughly washed twice with distilled water to remove castor oil and excess hairs. Sandfly samples were preserved in $1.5 \mathrm{~mL}$ Eppendorf tubes containing 70\% ethanol and drops of glycerin to prevent stiffen of sample and to avoid samples dry from alcohol. Then, labels indicating the site and day of sampling were attached. They were processed according to the methods described before (13). Samples shall be kept for a short period to prevent decomposition of samples. In the laboratory, the samples are washed thoroughly with distilled water. The samples were then placed in alcohol for an hour for removing water. The samples were then placed on glass slides. The samples should be well positioned to allow observing their taxonomic and morphological characteristics. External morphological analyses of the different species were carried out based on the sand fly mounted on each slide. Drops of mounting medium (DPX mountant) were added to avoid the occurrence of air bubbles. Then, the sample was left for a period of time to dry, and the cover slip was gently put on the specimen. These slides of sand fly were prepared for morphological identification using classification keys as described in a previous study (14).

Internal morphological analyses of different organ structures were performed using a light and dissecting microscope. Identification of sand flies was based on morphological structures; therefore, specimens were required to be prepared on microscopic slides (15).

\section{Molecular Study}

Primers

Two types of primers (Bioneer, Korea) were used for the diagnosis of Leishmania species using a nested PCR technique, which were designed in a previous study (2) as follows:

First primer F CGAGTAGCAGAAACTCCCGTTCA KDNA R ATTTTTCGCGATTTTCGCAGAACG 750bp

\section{Second primer F ACTGGGGGTTGGTGTAAAATAG KDNA R TCGCAGAACGCCCCT 560bp}

\section{Virulence Factors Primers (PCR Primers)}

The GP63 primers of leishmania spp. were used for the diagnosis of glycoprotein 63 gene in Leishmania spp. These primers are designed in this study by using a gene bank database NCBI and Primer3Plus software. 


\section{F GTACGTTACTCTCGACGCCG}

\section{R ATCACGAAGTCGGTGTTGCT 405bp}

\section{Molecular Diagnosis}

The PCR technique was used to detect Leishmania spp. and distinguish it from DNA samples of the insect. This method was implemented according to a previous study (16).

\section{DNA Extraction}

The gSYNC ${ }^{\mathrm{r}}$ DNA Extraction Kit is enhanced for genomic .The purified DNA is suitable for use in PCR or other enzymatic reactions. Genomic DNA from tissue samples were extracted by using gSYAN DNA mini kit extraction kit (Insect Protocol Procedure) Geneaid, USA, and done according to manufacturer's instructions.

The quality of the isolated genomic DNA was determined using a Nanodrop spectrophotometer (Thermo, USA), which assessed DNA concentration (ng/L) and DNA purity by reading the absorbance at $260 / 280 \mathrm{~nm}$.

\section{Preparation of Nested PCR Master Mix}

The Nested PCR master mix was prepared using Maxime PCR PreMix (iNtRON, Korea) according to the company instructions.

\section{First-Round PCR}

Nested PCR master mix for the first-round PCR was prepared according to the manufacturer's instructions by adding $5 \mu \mathrm{L}$ of genomic DNA, $1 \mu \mathrm{L}$ of first-round PCR product (10 pmol), $1 \mu \mathrm{L}$ of reverse primers (10 pmol), and $13 \mu \mathrm{L}$ of PCR water in a total volume of $20 \mu \mathrm{L}$.

In addition, the components of the above-mentioned PCR master mix reaction were lyophilized and stored in special PCR preMix tubes containing all additional PCR reaction components such as Taq polymerase DNA, Tris- $\mathrm{HCl}$ (pH: 9.0), dNTPs, $\mathrm{MgCl} 2, \mathrm{KCl}$, stabilizer, and tracking dye. Then, the tubes were centrifuged in an ExiSpin vortex centrifuge for 3 minutes and transferred to the PCR thermocycler.

\section{Primary Thermocycler Reaction Conditions}

PCR thermocycler conditions were set as follows (2): 1 cycle of initial denaturation at $94^{\circ} \mathrm{C}$ for 5 minutes, 30 cycles of denaturation at $95^{\circ} \mathrm{C}$ for 30 seconds, 30 cycles of annealing at $55^{\circ} \mathrm{C}$ for 30 seconds, 30 cycles of extension at $72^{\circ} \mathrm{C}$ for 30 seconds, and 1 cycle of final extension at $72^{\circ} \mathrm{C}$ for 5 minutes), then left at $4 \mathrm{C}^{\circ}$ for indefinite time.

\section{Second-Round Nested PCR}

Nested PCR master mix was prepared by adding $3 \mu \mathrm{L}$ of first-round PCR product, $1.5 \mu \mathrm{L}$ of forward primers (10 pmol), $1.5 \mu \mathrm{L}$ of reverse primers $(10 \mathrm{pmol})$, and $14 \mu \mathrm{L}$ of PCR water in a total volume of $20 \mu \mathrm{L}$. After the preparation of the polymerase chain reaction mixture was completed, the tubes were closed and mixed carefully for 10 seconds. Then, the tubes were transferred to a PCR thermocycler.

\section{Secondary Thermocycler Reaction Conditions}

PCR thermocycler conditions were set as follows:

One cycle of initial denaturation at $95^{\circ} \mathrm{C}$ for 5 minutes, 30 cycles of denaturation at $95^{\circ} \mathrm{C}$ for 30 seconds, 30 cycles of annealing at $55^{\circ} \mathrm{C}$ for 30 seconds, 30 cycles of extension at $72^{\circ} \mathrm{C}$ for 30 seconds, and 1 cycle of final extension at $72^{\circ} \mathrm{C}$ for 5 minutes). then left at $4 \mathrm{C}^{\circ}$ for indefinite time.

\section{Electrophoresis}

Nested PCR products at last steps were analyzed by loading in $1 \%$ Agarose to determine the presence or absence of PCR products as in the previous steps.

Detecting of the Virulence Factor GP63 of Leishmania in Sandfly

PCR technique was performed to detect virulence factor gene (gp63) of Leishmania spp. in DNA of positive cutaneous leishmaniasis samples of sand flies in the previous steps (nested PCR technique).

\section{Preparation of PCR Master Mix}

PCR master mix was prepared by adding $5 \mu \mathrm{L}$ of genomic DNA, $1 \mu \mathrm{L}$ of forward primers (10 pmol), $1 \mu \mathrm{L}$ of reverse primers $(10 \mathrm{pmol})$, and $13 \mu \mathrm{L}$ of PCR water in a total volume of $20 \mu \mathrm{L}$. After preparation, the above-mentioned PCR master mix reaction components were placed in standard PCR tubes containing the PCR PreMix as lyophilized material containing all additional PCR reaction components such as Taq DNA polymerase, Tris- $\mathrm{HCl}$ (pH: 9.0), dNTPs, $\mathrm{MgCl} 2, \mathrm{KCl}$, stabilizer, and tracking dye.

Then, the tubes were centrifuged for 3 minutes in an ExiSpin vortex centrifuge and put in PCR thermocycler.

\section{PCR Thermocycler Reaction Conditions}

PCR thermocycler reaction conditions were as follows: 1 cycle of initial denaturation at $95^{\circ} \mathrm{C}$ for 5 minutes, 30 cycles of denaturation at $95^{\circ} \mathrm{C}$ for 30 seconds, 30 cycles of annealing at $55^{\circ} \mathrm{C}$ for 30 seconds, 30 cycles of extension at $72^{\circ} \mathrm{C}$ for 1 minute, and $1 \mathrm{cycle}$ of final extension at $72^{\circ} \mathrm{C}$ for 5 minutes. Then left at $4^{\circ} \mathrm{C}$ for indefinite time.

\section{Electrophoresis}

In the last step, Nested PCR products were analyzed by loading in $1 \%$ Agarose gel to determine the presence or absence of PCR products as in previous steps.

\section{Results and Discussion}

Molecular Diagnostics of Leishmania Inside the Insect Using Nested PCR

Twenty-five samples were used to detect the parasite causing the disease in the study area. The results of the gel 
electrophoresis indicated that the DNA samples extracted from the insects collected from the study areas infected with Leishmania. Special primers for the L. major and L. tropica species were used for kinetoplast DNA gene (kDNA), with Nested PCR product of 560 bp length for $L$. major as well as $750 \mathrm{bp}$ for L. tropica.

Based on the results of the gel electrophoresis, 13 samples were positive, containing DNA for the parasite including L. major and L. tropica species (52\%), and 12 samples were negative for both species (48\%). Positive samples included 11 samples of $L$. major species (84.6\%) and only 2 samples of $L$. tropica species (15.3\%). The results show that rural species of $L$. major was the dominant species in the study area. Figure 1 shows the appearance of DNA bundles of Leishmania species found in the study area.

A nested-PCR-based approach allows the detection of Leishmania directly from samples (12). It was reported that nested PCR is a reliable method for diagnosing and identifying Leishmania species that can be used in epidemiologic studies. With the correct primers, it can be used to distinguish between species (13).

It was mentioned that nested PCR entails the use of two sets of primers in two separate cycles. A secondary target within the first product is amplified by the second pair of primers. This eliminates contamination from primer dimers, hairpins, and alternate primer targets in the second PCR result. This study employed an approach similar to that utilized by Akhavan et a1 (13) in Iran.

The PCR methodology was utilized to diagnose $L$. major (560 bp) gene as well as L. tropica (750 bp) gene.

\section{Molecular Diagnosis of the Virulence Factor Glycoprotein 63 (gp63) Gene in Promastigote Form of Leishmania Samples from Sandflies Using PCR}

The DNA of positive samples of sand flies including 11 samples of $L$. major species and 2 samples of $L$. tropica species was used for diagnosis of virulence gene (GP63) on the surface of promastigote form of Leishmania from insect using PCR technique. The results show that the parasite contains virulence factor gene (Gp63) on the surface of promastigote. Figure 2 shows the appearance of bundles of virulence factor gene (Gp63) in cutaneous leishmaniasis positive samples in the study region.

The Leishmania genus has developed several ways for inhibiting the innate immune response, allowing infection and spread within the macrophages of the mammalian host.

Several virulence factors of Leishmania have been discovered to have a role in the progression of leishmaniasis.

Recent discoveries, on the other hand, highlight the significance of GP63 as an avirulence factor that has a significant impact on signaling processes and activities of host cells. The results corroborate previous findings.

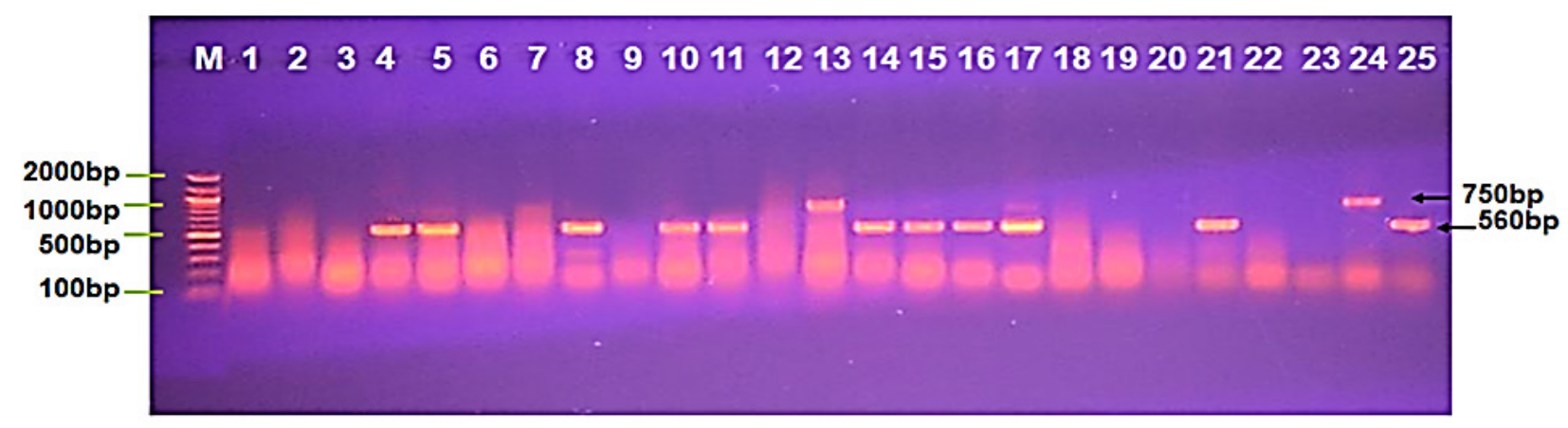

Figure 1. Image of Agarose Gel Electrophoresis of DNA fragment Stained with Ethidium Bromide.

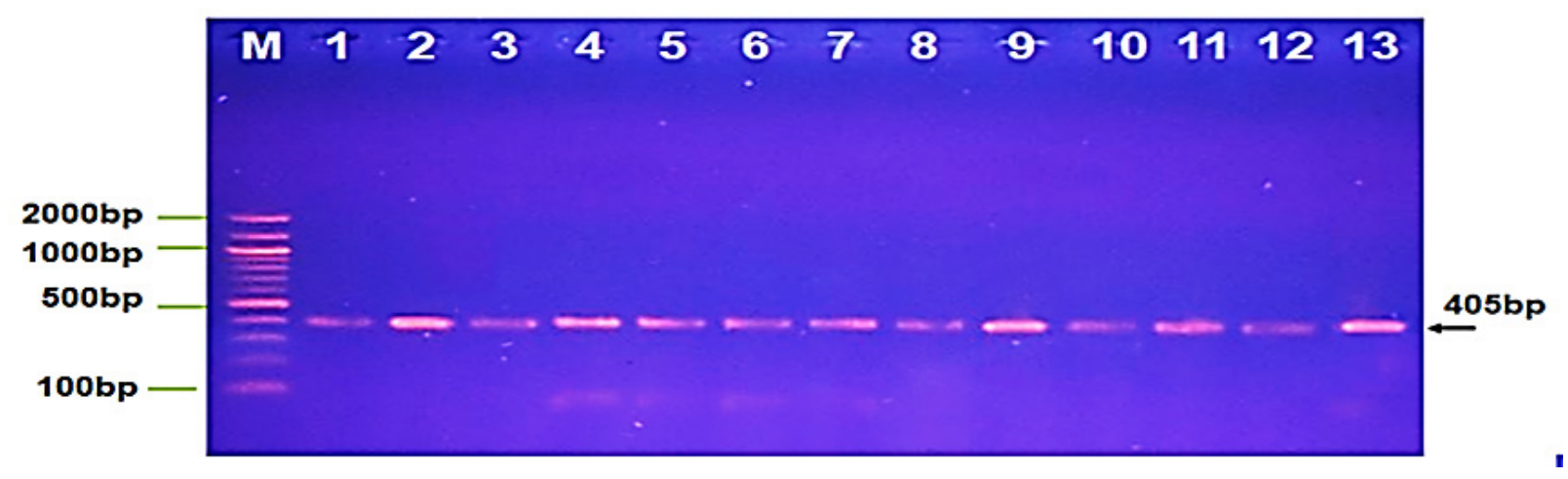

Figure 2. Image of Agarose Gel Electrophoresis showing the PCR Product Analysis of Virulence Factor Gene (Gp63) in Cutaneous Leishmaniasis Positive Samples. 
Glycoprotein 63, known as leishmanolysin, is a zincdependent metalloprotease that has been demonstrated to promote phagocytosis and macrophage survival by mediating entry into macrophages (15).

The biological activity of parasites is linked to their ability to shield themselves from host enzymes in insect vector midguts and macrophage phagolysosomes (16). The GP63 genes are found in both promastigotes and amastigotes, and their products play a role in parasite attachment and macrophage internalization. GP63 is also used by Leishmania species to move across the extracellular matrix, avoid lysis by inactivating complement system components, and hydrolyze intracellular macrophage targets. Leishmania uses this method to survive and spread within mammalian host cells. A greater understanding of the processes by which this virus is able to evade the innate immune response may aid in the development of new anti-Leishmania therapies (17).

Glycoprotein 63 (gp63) or leishmanolysin, which is a 63 $\mathrm{kDa}$ protein and a metalloprotease, is a virulence factor that interacts directly with host macrophage receptors as well as with the complement system. The role of GP63 in a vector is yet unknown.

The enzyme could help with nutrition acquisition and protect promastigotes from digestive enzymes in the midgut. Sand is a substance that can be used in a variety of ways. The midgut protease activity of sandflies appears to peak one to three days afterward.

Gp63 is a virulence factor that plays a range of roles in host-parasite interactions. Expression of a cloned leishmanolysin gene, which has been proposed as a virulence factor, could considerably amplify the phenotypic differences.

These results suggest that leishmanolysin is involved in Leishmania pathogenesis (18). Leishmania spp., which reside as extracellular promastigotes in the Sand Fly and internal amastigotes in macrophages' phagolysosome compartment, have been discovered to include (gp63). The importance of gp63 in the Leishmania vector is less well understood.

Gp63 is a virulence factor that plays a range of roles in host-parasite interactions. The importance of gp63 in Leishmania vector is less well understood. Gp63 is thought to break down hemoglobin and other proteins of the blood in the meals, providing nourishment for promastigotes to reproduce. It also interacts with the complement system, which helps amastigote form of Leishmania spp. survive inside macrophages $(8,19)$.

This is the first study conducted in Iraq which used the virulence factors gene Leishmanolysin (glycoprotein 63) or Leishmania metalloproteinase to detect Leishmania in sand flies.

\section{Conclusion}

Three species of sand flies are found in Al-Muthanna province, two species of Phlebotomus and one species of Sergentomyia (Phlebotomus papatasi, Phlebotomus sergenti, and Sergentomyia sintoni, respectively ). $P$. papatasi is the most abundant species in the province and possibly the main carrier of Leishmania.

This study revealed the possibility of diagnosing leishmaniasis by virulence gene (gp63) in Leishmania promastigotes.

\section{Conflict of Interests}

The authors declared that no competing interests exist.

\section{Ethical Issues}

In this research, ethical considerations have been fully observed.

\section{Acknowledgement}

The authors would like to acknowledge all staff of Leishmaniasis Unit and Ecology Department in Al-Muthana province for their support and thank the all those who helped.

\section{Authors' Contributions}

We did writing, editing of the manuscript, statistical analysis, and data collection.

\section{References}

1. Olivier M, Atayde VD, Isnard A, Hassani K, Shio MT. Leishmania virulence factors: focus on the metalloprotease GP63. Microbes Infect. 2012;14(15):1377-1389. doi:10.1016/j.micinf.2012.05.014

2. Noyes HA, Reyburn H, Bailey JW, Smith D. A nested-PCRbased schizodeme method for identifying Leishmania kinetoplast minicircle classes directly from clinical samples and its application to the study of the epidemiology of Leishmania tropica in Pakistan. J Clin Microbiol. 1998;36(10):2877-2881. doi:10.1128/jcm.36.10.28772881.1998

3. Maroli M, Feliciangeli MD, Bichaud L, Charrel RN, Gradoni L. Phlebotomine sandflies and the spreading of leishmaniases and other diseases of public health concern. Med Vet Entomol. 2013;27(2):123-147. doi:10.1111/j.13652915.2012.01034.x

4. Lane RP. Sandflies (Phlebotominae). In: Lane RP, Crosskey RW, eds. Medical Insects and Arachnids. Dordrecht: Springer; 1993:78-119. doi:10.1007/978-94-011-15544_4pp. 78-119

5. Walker DM, Oghumu S, Gupta G, McGwire BS, Drew ME, Satoskar AR. Mechanisms of cellular invasion by intracellular parasites. Cell Mol Life Sci. 2014;71(7):12451263. doi:10.1007/s00018-013-1491-1

6. Isnard A, Shio MT, Olivier M. Impact of Leishmania metalloprotease GP63 on macrophage signaling. Front Cell Infect Microbiol. 2012;2:72. doi:10.3389/fcimb.2012.00072

7. Chang KP, McGwire BS. Molecular determinants and regulation of Leishmania virulence. Kinetoplastid Biol Dis. 2002;1(1):1. doi:10.1186/1475-9292-1-1

8. Yao C, Donelson JE, Wilson ME. The major surface protease (MSP or GP63) of Leishmania sp. Biosynthesis, regulation of expression, and function. Mol Biochem Parasitol. 2003;132(1):1-16. doi:10.1016/s0166-6851(03)00211-1 
9. Voth BR, Kelly BL, Joshi PB, Ivens AC, McMaster WR. Differentially expressed Leishmania major GP63 genes encode cell surface leishmanolysin with distinct signals for glycosylphosphatidylinositol attachment. Mol Biochem Parasitol. 1998;93(1):31-41. doi:10.1016/s01666851(98)00013-9

10. Al-Mayali HMH. Evaluation and use of some immunological tests in the epidemiological study of leishmaniasis in Qadisiyah province (thesis). Dīwānīyah, Iraq: University of Al-Qadisiyah; 2004.

11. Abul-Hab J, Ahmed SA. Revision of the family Phlebotomidae (Diptera) in Iraq. Vol. 7. Baghdad, Iraq: Journal of Biological Sciences Research; 1984;7:1-37.

12. Akhavan AA, Mirhendi $\mathrm{H}$, Khamesipour A, et al. Leishmania species: detection and identification by nested PCR assay from skin samples of rodent reservoirs. Exp Parasitol. 2010;126(4):552-556. doi:10.1016/j. exppara.2010.06.003

13. Akhavan AA, Mirhendi $\mathrm{H}$, Khamesipour A, et al. Leishmania species: detection and identification by nested PCR assay from skin samples of rodent reservoirs. Exp Parasitol. 2010;126(4):552-556. doi:10.1016/j. exppara.2010.06.003

14. Contreras I, Gómez MA, Nguyen O, Shio MT, McMaster RW, Olivier M. Leishmania-induced inactivation of the macrophage transcription factor AP-1 is mediated by the parasite metalloprotease GP63. PLoS Pathog. 2010;6(10):e1001148. doi:10.1371/journal.ppat.1001148

15. Russell DG. The macrophage-attachment glycoprotein GP63 is the predominant C3-acceptor site on Leishmania mexicana promastigotes. Eur J Biochem. 1987;164(1):213221. doi:10.1111/j.1432-1033.1987.tb11013.x

16. Joshi PB, Kelly BL, Kamhawi S, Sacks DL, McMaster WR. Targeted gene deletion in Leishmania major identifies leishmanolysin (GP63) as a virulence factor. Mol Biochem Parasitol. 2002;120(1):33-40. doi:10.1016/s01666851(01)00432-7

17. Chaudhuri G, Chaudhuri M, Pan A, Chang KP. Surface acid proteinase (GP63) of Leishmania mexicana. A metalloenzyme capable of protecting liposomeencapsulated proteins from phagolysosomal degradation by macrophages. J Biol Chem. 1989;264(13):7483-7489.

18. Ilgoutz SC, McConville MJ. Function and assembly of the Leishmania surface coat. Int J Parasitol. 2001;31(9):899908. doi:10.1016/s0020-7519(01)00197-7

19. Chang KP, Reed SG, McGwire BS, Soong L. Leishmania model for microbial virulence: the relevance of parasite multiplication and pathoantigenicity. Acta Trop. 2003;85(3):375-390. doi:10.1016/s0001-706x(02)00238-3

(C) 2020 The Author(s); This is an open-access article distributed under the terms of the Creative Commons Attribution License (http:// creativecommons.org/licenses/by/4.0), which permits unrestricted use, distribution, and reproduction in any medium, provided the original work is properly cited. 